A.

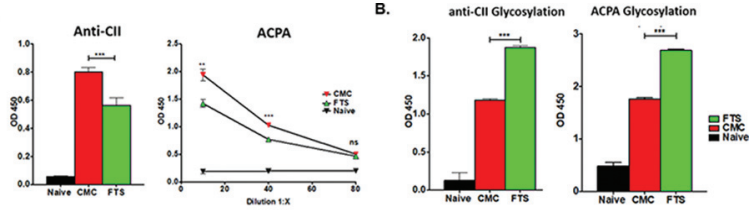

Acknowledgement: This work was supported by the U.S Department of Defense Grant number AMRMC W81XWH-14-1-0609/10

Disclosure of Interests: None declared

DOI: 10.1136/annrheumdis-2019-eular.4548

\section{SAT0045 A NOVEL SMALL MOLECULE, MBS2133, MODULATES OSTEOCLAST PRE-CURSOR METABOLISM TO INHIBIT OSTEOCLAST DIFFERENTIATION: AN ALTERNATIVE THERAPY FOR OSTEOLYTIC PATHOLOGY IN RA}

Shatakshi Sood ${ }^{1}$, Lisa Patel ${ }^{2}$, Martyn Foster ${ }^{2}$, Louise Jopling ${ }^{3}$, Rob Van't Hof ${ }^{4}$, lain Greig ${ }^{5}$, Sam Williams ${ }^{2}$, lain Mcinnes ${ }^{1}$, Carl Goodyear'. ${ }^{1}$ University of Glasgow, Institute of Infection, Immunity and Inflammation, Glasgow, United Kingdom; ${ }^{2}$ Istesso Ltd, London, United Kingdom; ${ }^{3} J o h n s o n$ and Johnson Innovation, London, United Kingdom; ${ }^{4}$ University of Liverpool, Institute of Ageing and Chronic Disease, Liverpool, United Kingdom; ${ }^{5}$ University of Aberdeen, Institute of Medical Sciences, Aberdeen, United Kingdom

Background: Rheumatoid arthritis (RA) is a chronic inflammatory disease associated with substantial local and systemic bone loss. Despite the availability of several treatment options many patients do not reach low disease activity. Furthermore, current therapeutics generally target inflammation rather than erosive pathology. Thus, there remains a need for new therapies that can target both aspects of the disease. Prior studies have shown that biphenylcarboxylic acid small molecule derivatives no only inhibit murine osteoclastogenesis but also attenuate inflammation and bone destruction in murine models of $\mathrm{RA}^{1,2}$

Objectives: To evaluate a novel small molecule derivative, MBS2133, on human osteoblastogenesis, osteoclastogenesis and cellular function, and to investigate the in vitro mechanism-of-action.

Methods: Osteoblasts were derived from human mesenchymal stem cells. Cells were differentiated in the presence or absence of MBS2133 and mineralization assessed by Alizarin Red staining. Human $\mathrm{CD}_{14}{ }^{+}$blood monocytes were differentiated into osteoclasts (OCs) with M-CSF and RANK-L, in the presence or absence of MBS2133, and/or metabolites. Mature OCs were stained with tartrate-resistant acid phosphatase (TRAP) and quantified by light microscopy. Osteolytic activity was assessed on mineral-coated surfaces. Western blot analysis was used to assess downstream signalling pathways. Changes in the metabolic profile of pre-osteoclasts following 4h exposure to MBS2133 was carried out by liquid chromatography mass spectrometry.

Results: MBS2133 had no effect on the differentiation and function of primary human osteoblasts. In comparison, exposure of RANK-L stimulated $\mathrm{CD}_{14}{ }^{+}$monocytes to MBS2133 significantly reduced OC differentiation and osteolytic activity of mature OCs. Notably, exposure of pre-OCs to MBS2133 for $2 \mathrm{~h}$ at initiation of osteoclastogenesis, was sufficient to significantly reduce subsequent $O C$ differentiation. Evaluation of treated preosteoclasts revealed that RANKL-mediated phosphorylation of p38 was reduced. Metabolomic analysis of pre-osteoclasts revealed that MBS2133 induced a substantial reduction in a range of metabolites associated with glycolysis, oxidative phosphorylation and fatty acid oxidation pathway. Notably, L-carnitine, which facilitates the transportation of fatty acids to the mitochondrial matrix and enables processing and entry into tricarboxylic acid (TCA) cycle for further energy production, was significantly reduced. In vitro supplementation of L-carnitine inhibited the ability of the compound to switch off OC differentiation and osteolytic activity.

Conclusion: The results of this study demonstrate that MBS2133 specifically modulates the metabolome of myeloid cells, which has a substantia impact on their ability to differentiate into mature osteoclasts. These findings highlight the importance of modulating the glycolysis/oxidative phosphorylation axis in osteoclastogenesis and suggest that targeting the metabolic state of pre-osteoclasts could offer a new therapeutic approach to treat bone resorption in rheumatic diseases.

\section{REFERENCES}

[1] Greig IR, et al. Development and Characterization of Biphenylsulfonamides as Novel Inhibitors of Bone Resorption. J.Med.Chem. 2006:49:7487-7492

[2] Coste $E$, et al. Identification of small molecule inhibitors of RANKL and TNF signalling as anti-inflammatory and antiresorptive agents in mice. AnnRheumDis 2015;74:220-6.
Disclosure of Interests: Shatakshi Sood: None declared, Lisa Patel Shareholder of: Shareholder of Istesso Ltd. Employee of: Employee of Istesso, Martyn Foster Shareholder of: AstraZeneca, Consultant for: Istesso, Levicept, Employee of: AstraZeneca, Louise Jopling Shareholder of: Johnson and Johnson (employee), Employee of: Employee of Janssen (Pharmaceutical arm of Johnson \& Johnson) since May 2008 to present day, Rob van't Hof Shareholder of: OsteoRx Ltd, lain Greig Shareholder of: Shareholder in OsteoRx Ltd, a spin-out company from the University of Aberdeen, which retains a financial interest in MBS2133, Sam Williams Shareholder of: Shareholders of Istesso Ltd, Employee of: Employees of Istesso, lain McInnes Grant/research support from: AstraZeneca, Celgene, Compugen, Novartis, Roche, UCB Pharma, Consultant for: AbbVie, Celgene, Galvani, Lilly, Novartis, Pfizer, UCB Pharma, Carl Goodyear Grant/ research support from: AstraZeneca, BMS, Celgene, Janssen, MedAnnex, Pfizer and UCB, Speakers bureau: Abbvie DOI: 10.1136/annrheumdis-2019-eular.5482

\section{SAT0046 ARE SENSE OF SOCIAL SUPPORT AND LOW DECISION LATITUDE AT WORK LINKED TO RISK OF RHEUMATOID ARTHRITIS, AND IF SO, HOW DO THEY RELATE TO OTHER RISK FACTORS? RESULTS FROM THE SWEDISH EIRA STUDY}

Louise Hedenstierna ${ }^{1,2}$, Christina H. Opava ${ }^{3}$, Sofia Ernestam² , Johan Askling $^{4}$, Lars Klareskog ${ }^{4}$, Lars Alfredsson ${ }^{1}$, Saedis Saevarsdottir ${ }^{1} .{ }^{1}$ Environmental Medicine, Karolinska Institutet, Stockholm, Unit of translational epidemiology, Stockholm, Sweden; ${ }^{2}$ Centrum for rheumathology, Stockholm, Sweden; ${ }^{3}$ Department of Neurobiology, Care Sciences and Society, Karolinska Institutet, Unit of physiotherapy, Stockholm, Sweden; ${ }^{4}$ Karolinska Institutet, Stockholm, Sweden, Rheumatology unit, Department of medicine, Stockholm, Sweden

Background: The role of psycho-social factors in the development of rheumatoid arthritis (RA) is debated.

Objectives: We investigated whether psychosocial stress measured as low sense of social support, and low decision latitude at work, were linked to risk of RA, and whether they related to known lifestyle risk factors for RA.

Methods: The Swedish population-based EIRA study included incident RA cases $(\mathrm{N}=3724)$ and controls $(\mathrm{N}=5937)$, matched for age, sex and residential area. Responders filled in questionnaires regarding self-reported social support, decision latitude at work and life-style-factors.

The distribution of answers among controls were used to define exposure, thus for social support, those in the lowest quartile of social support were considered exposed to low social support and similarly for decision latitude, those in the lowest quartile were considered exposed to low decision latitude at work.

Using logistic regression, we first evaluated whether exposures associated with RA risk, considering potential confounding of established risk factors. Then, we investigated whether the frequency of those factors differed between individuals reporting low social support or low decision latitude at work or not, among cases and controls.

Results: There were 898 cases with low social support and 285 cases with low decision latitude at work (latter only available in first part of EIRA).

Low social support was not associated with RA risk in unadjusted analyses $(O R=1.05,95 \% \mathrm{Cl}=0.95-1.15)$. Low decision latitude at work did associate with a higher RA risk in the unadjusted analyses $(\mathrm{OR}=1.52$, $95 \% \mathrm{Cl}=1.20-1.94)$, but this association was no longer significant after further adjustment for smoking, obesity and university degree (adjusted $\mathrm{OR}=1.24,95 \% \mathrm{Cl}=0.93-1.63)$. Associations between those life-style risk factors and RA were confirmed (no university degree, $O R=1.50$; smoking $\mathrm{OR}=1.71$; obesity $\mathrm{OR}=1.15$ )

Next, we evaluated whether low social support or low decision latitude at work differed by previously established risk factors. Cases with RA reporting low sense of social support were more often men $(\mathrm{OR}=1.60,95 \%$ $\mathrm{Cl}=1.40-1.83)$, current smokers $(\mathrm{OR}=1.46, \quad 95 \% \mathrm{Cl}=1.26-1.70)$, obese $(\mathrm{OR}=1.29,95 \% \mathrm{Cl}=1.09-1.54)$, physically inactive $(\mathrm{OR}=2.78,95 \% \mathrm{Cl}=1.98$ $3.90)$ and without a university-degree $(\mathrm{OR}=2.04,95 \% \mathrm{Cl}=1.77-2.36)$; with similar pattern among the controls. For working-conditions, cases reporting low decision latitude at work were also more often current smokers $(\mathrm{OR}=2.05,95 \% \mathrm{Cl}=1.33-3.16)$ and with no university degree $(\mathrm{OR}=8.23$, $95 \% \mathrm{Cl}=5.13-13.22)$, but less often male $(\mathrm{OR}=0.40,95 \% \mathrm{Cl}=0.26-0.60)$ Again, the pattern was similar among controls. RF/ACPA-positivity did not associate with low social support or low decision latitude.

Conclusion: Neither low social support nor low decision latitude at work were associated with an increased risk of RA after adjustment for other known lifestyle risk factors for RA. An initial crude association between 
low decision latitude at work and risk for RA was explained by differences in smoking and educational level. However, both low social support and low decision latitude at work associate strongly with known, and here validated, risk factors for RA (smoking, obesity, no university degree) with similar pattern among both cases and controls.

Acknowledgement: The EIRA study group

Disclosure of Interests: Louise Hedenstierna: None declared, Christina $\mathrm{H}$. Opava: None declared, Sofia Ernestam: None declared, Johan Askling Grant/research support from: Karolinska Institutet (JA) has or has had research agreements with the following pharmaceutical companies, mainly in the context of the ATRIS national safety monitoring programme for rheumatology biologicals: Abbvie, BMS, MSD, Eli Lilly, Pfizer, Roche, Samsung Bioepis, and UCB., Consultant for: Karolinska Institutet has received remuneration for JA participating in ad boards arranged by Lilly, Novartis, and Pfizer., Lars Klareskog Grant/research support from: Yes, but not for the presented study., Lars Alfredsson: None declared, Saedis Saevarsdottir Employee of: Part-time employee at deCODE Genetics/ Amgen Inc, working on genetic research unrelated to this project. DOI: 10.1136/annrheumdis-2019-eular.6088

\section{SAT0047 PRIMARY LYMPHOID TISSUE FROM RHEUMATOID ARTHRITIS PATIENTS HARBOUR CITRULLINE SPECIFIC PLASMA CELLS}

Radha Thyagarajan ${ }^{1}$, Aase Hensvold ${ }^{1,2}$, Lena Israelsson ${ }^{1}$, Johanna Steen ${ }^{1}$ Heidi Wähämaa ${ }^{1}$, Annika van Vollenhoven ${ }^{1}$, Khaled Amara ${ }^{1}$, Anca Catrina ${ }^{1,2}$ Vivianne Malmström ${ }^{1}$, Caroline Grönwall ${ }^{1} .{ }^{1}$ Rheumatology unit Karolinska University Hospital and Karolinska Institutet, Stockholm, Sweden; ${ }^{2}$ Center for Rheumatology, Academic Specialist Center, Stockholm Health Services, Stockholm, Sweden

Background: Anti-citrullinated protein antibodies (ACPA) are specific markers with pathological effects in rheumatoid arthritis (RA). ACPA-specific B-cells have been identified in synovial joint fluid and peripheral blood. An increased concentration of ACPA is found in synovial fluid and lung tissue compared to blood suggest local production, partly confirmed by detection ACPA synovial plasma blasts, but primary lymphoid tissue have not previously been examined.

Objectives: In this study we investigated the plasma cell repertoire in the bone marrow and occurrence of autoantibody producing plasma cells. Methods: For this study we developed a method to collected and process bone marrow samples from proximal femur in RA patients undergoing hip joint replacement. Bone marrow samples were processed and mononuclear cells were obtained by Ficoll separation. CD138+ plasma cells were single cell sorted by flow cytometry. Paired heavy and light chains were PCR amplified, sequenced, and analyzed by V-Quest and IgBLAST towards the IMGT database to annotate variable gene usage. To enrich for ACPA, sequences were selected based on high somatic hypermutations number and Fab N-glycosylation sites. Selected sequences were cloned and expressed as IgG in Expi293 cells. Monoclonal antibody reactivity to citrullinated and arginine form of vimentin, enolase, fibrinogen, histone, tenascin C peptides; carbamylated and malondialdehyde-acetaldehyde bovine serum albumin, and tetanus toxoid antigens were examined by ELISA. Included patients were clinical examined and donating peripheral blood samples.

Results: After method development and quality analysis we were able to include five bone marrow samples in this study. The five included RA patients, median age 74 (range 66-82) years, had in median 20 (range 4-44) years of destructive disease, all were RF positive, four were females and anti-CCP2-positive, and all except one were treated with anti-rheumatic drugs.

Overall, from the processed bone marrow, 465 paired heavy-light IgG plasma cell sequences were obtained, in total 368 (range 20-194) from ACPA positive patients and 97 from the ACPA negative patient. We observed statistically significant changes in heavy chain variable gene usage with lower $\mathrm{VH}-1$ and higher $\mathrm{VH}-3$ frequency in sequences from ACPA positive RA patients compared to sequences from the ACPA negative RA patient. We also found statistically significant increase in $\mathrm{VH} N-$ glycosylation in sequences from the ACPA positive RA patients $(22.6 \%$ ACPA positive vs $12.4 \%$ ACPA negative; $p=0.03$ ) but no difference in mutation numbers. From obtained sequences, 34 interesting clones were selected for monoclonal antibody-expression. Among the 34 clones we found two citrulline specific reactive clones (reactivity towards CCP2, citrullinated vimentin and fibrinogen peptides) and one malondialdehyde-acetaldehyde (MAA) reactive clone, originating from three different APCA positive patients with the most longstanding disease. None of these clones had reactivity towards control antigens such as arginine versions of peptides.

Conclusion: Plasma cells producing ACPA are present in primary lymphoid tissue from RA patients. Further studies of difference in characteristics, IgG gene usage and $\mathrm{N}$-glycosylation, between ACPA-positive and ACPA-negative patients are needed.

Disclosure of Interests: Radha Thyagarajan: None declared, Aase Hens vold: None declared, Lena Israelsson: None declared, Johanna Steen: None declared, Heidi Wähämaa: None declared, Annika van Vollenhoven: None declared, Khaled Amara: None declared, Anca Catrina Grant/ research support from: Yes, but not for the presented study., Vivianne Malmström: None declared, Caroline Grönwall: None declared DOI: 10.1136/annrheumdis-2019-eular.5961

\section{SAT0048 1 ANTI-FRACTALKINE MONOCLONAL ANTIBODY AMELIORATE JOINT DESTRUCTION AND SYNOVIUM THROUGH SUPPRESSION OF OSTEOCLAST PRECURSOR MIGRATION AND INDUCTION OF SYNOVIAL CELL DEATH IN COLLAGEN-INDUCED ARTHRITIS MODEL}

Naoto Ishii ${ }^{1}$, Kana Hoshino-Negishi ${ }^{1}$, Masayoshi Ohkuro ${ }^{2}$, Tomoya Nakatani ${ }^{1}$, Wataru Ikeda ${ }^{1}$, Yoshikazu Kuboi ${ }^{1}$, Nobuyuki Yasuda ${ }^{1}$, Toshio Imai ${ }^{1}{ }^{1}$ KAN Research Institute Inc., Kobe, Japan; ${ }^{2}$ EA Pharma Co., Ltd., Kawasaki, Japan

Background: In the Phase 1/2 clinical study, E6011, a novel humanized anti-fractalkine $(F K N)$ mAb demonstrated a promising efficacy in active RA patients who were inadequately controlled by MTX and/or TNF- $\alpha$ inhibitors (NCT 02196558) $)^{1}$.In RA joint tissue, increased expression of fractalkine (FKN) and abundant infiltration of CX3CR1-positive cells were observed $^{2}$. FKN is expressed on endothelial cells and fibroblast-like synoviocytes in synovium and also expressed on osteoblasts. CX3CR1 is expressed on monocytes/macrophages and osteoclast precursors (OCPs). Therefore, FKN-CX3CR1 interaction could play pivotal roles in migration, differentiation and activation of those cells. However, the precise mechanism(s) of FKN-CX3CR1 axis in RA, especially on joint destruction remains to be elucidated.

Objectives: To elucidate the roles of FKN-CX3CR1 axis in cartilage destruction, bone damage and proliferated synovial cells by using antimouse FKN mAb (anti-mFKN mAb).

Methods: For the induction of $\mathrm{ClA}$, mice were immunized with bovine type II collagen. Anti-mFKN mAb was injected twice a week. The clinical arthritis score was monitored, and joint destruction was evaluated by soft $x$-ray and histology. The mRNA expression levels were assessed by quantitative RT-PCR. Blood parameters were measured using ELISA. In in vitro, effect of immobilized FKN on RANK ligand (RANKL)-induced osteoclast differentiation was examined. In in vivo, bone marrow-derived OCPs were fluorescein-labeled and transferred to $\mathrm{ClA}$ mice to evaluate the migration of OCPs into synovium. Inhibitory effect of anti-mFKN mAb, etanercept or tofacitinib against OCP migration was assessed. To examine the effect of anti-mFKN mAb against proliferated synovial cells, propidium iodide $(\mathrm{PI})$ was injected to anti-mFKN mAb-treated CIA mice to detect the synovial cell death.

Results: Anti-mFKN mAb significantly reduced the arthritis and soft x-ray scores in both prophylactic and therapeutic treatment. Anti-mFKN mAb histologically improved synovitis, cartilage destruction and bone damage with marked reduction of osteoclast numbers. Plasma levels of COMP and MMP-3 were also decreased. Interestingly, anti-mFKN mAb significantly suppressed Tnf and II6 mRNA expression in the affected joints. In in vitro, RANKL-induced osteoclast differentiation was enhanced by immobilized FKN, and anti-mFKN mAb suppressed FKN-dependent osteoclas formation. In in vivo, anti-mFKN mAb strongly inhibited the migration of OCPs into the proliferated synovial cells of mice with CIA, whereas etanercept or tofacitinib had no significantly effect. Importantly, synovial cell death was abundantly found in proliferated synovial cells of mice with $\mathrm{CIA}$ after the anti-mFKN mAb treatment ${ }^{3}$.

Conclusion: Anti-mFKN mAb remarkably ameliorated the joint destruction with the marked reduction of osteoclasts by the inhibition of both OCP survival and OCP migration in inflamed joint tissues. In addition, antimFKN mAb immediately induced synovial cell death in the proliferated synovial cells, suggesting the direct inhibitory effect in the synovitis. These results strongly indicate that inhibition of FKN-CX3CR1 axis by a humanized anti-FKN mAb, E6011, is an attractive and affected jointsselective therapeutic strategy for the treatment of both inflammatory synovitis, cartilage destruction and bone damage in RA patients.

\section{REFERENCES}

[1] Tanaka Y, et al., Mod Rheumatol (2018) 28, 58-65 